\title{
ON CALCULATION OF THE COSMOLOGICAL PARAMETERS IN EXACT MODELS OF INFLATION.
}

\author{
S. V. Chervon ${ }^{1}$ and I. V. Fomin ${ }^{2}$ \\ Ulyanovsk, Ulyanovsk State University
}

Received October 31, 2007

\begin{abstract}
We discuss a method of calculating the key cosmological parameters on the basis of a selected scale factor by using exact solutions of the background equations. We specify the formulas for calculating the power spectrum, the spectral indices and their ratios, and the ratio of squared amplitudes of scalar and tensor perturbations. We obtain, for the first time, expressions for the basic cosmological parameters in terms of the scalar factor value at the instant of crossing the cosmological horizon. In this case, the cosmological parameters are calculated for a wide class of exact models. They are compared with their analogs derived in the slow-rolling approximation.
\end{abstract}

PACS: $98.80 .-\mathrm{k}, 98.80 \mathrm{Cq}$

\section{Introduction.}

The progress achieved in inflationary cosmology [1] enables us to compare the observational data with predictions of the theory: having chosen a cosmological model describing the Universe at its inflationary stage, one can determine the power spectrum of density perturbations, the spectral indices of scalar and tensor perturbations and their ratio as well as the ratio of squared amplitudes of the tensor and scalar modes. The basic theoretical conclusions have been obtained, as a rule, in the framework of the slow-roll approximation [3. A method of obtaining new theoretical conclusions on the basis of exact solutions was proposed comparatively recently [4, and this method has enabled us to introduce corrections to calculations of the perturbation spectrum at horizon crossing. An exact expression for calculating the spectral index in terms of the total energy of the scalar field was found in [6] for models of power-law and exponentialpower-law inflation. It was shown [7] how to obtain the key cosmological parameters at horizon crossing on the basis of a selected scale factor or, which is the same, the Hubble parameter, in the spirit of the potential fine tuning method [8,9] (see also [10-12]). The cosmological parameters were calculated and compared with their analogs ob-

\footnotetext{
${ }^{1}$ e-mail: sv chervon@rambler.ru

${ }^{2}$ e-mail: ingvor@inbox.ru
}

tained in the slow-rolling approximation for powerlaw and exponential-power-law inflation and for singular and nonsingular representations of de Sitter cosmology.

In this paper, we widen the circle of the exact models studied and discuss the possibility of recalculating the cosmological parameters at crossing the horizon for comparison with the observational data.

\section{The method of calculating the cosmological parameters}

Initial perturbations of a cosmological model of inflation are described by a self-consistent set of the Einstein equations and those of a self-interacting scalar field in Friedman-Robertson-Walker spacetime with flat spatial sections. Let us choose a presentation of this system in terms of the total energy potential $W(\phi)$ [13]:

$$
\begin{aligned}
W(\phi) & =3 M_{P}^{2} H^{2}, \\
3 H \dot{\phi} & =-\frac{d}{d \phi} W(\phi), \\
{[\dot{\phi}(t)]^{2} } & =-2 M_{P}^{2} \dot{H},
\end{aligned}
$$

Recalled that the total energy potential has been defined as a function of the scalar field

$$
W(\phi)=V(\phi)+\frac{1}{2} U^{2}(\phi), \quad U(\phi)=\dot{\phi}
$$


It is easy to verify that one of the Einstein equations (3) may be obtained as a consequence of Eqs. (1) and (2).

Taking into account an exact formula for differential of the logarithm of the wave vector at horizon crossing time, $k=a H$, valid for perturbations with the wave vector $k[6$,

$$
d(\ln k)=H d t+\frac{\dot{H}}{H} d t=H d t+\frac{3 U^{3}(\phi)}{2 M_{P}^{2} W^{\prime}} d t,
$$

one can make an amendment in the expression for the curvature perturbations power spectrum by substituting the physical potential $V(\phi)$ with the total energy potential $W(\phi)$; then, using Eqs. (1)(3), we can obtain a representation in terms of the Hubble parameter:

$$
\mathcal{P}_{\mathcal{R}}(k)=\frac{1}{12 \pi^{2} M_{P}^{6}} \frac{W^{3}}{W^{\prime 2}}=-\left.\frac{H^{4}}{8 M_{p}^{2} \dot{H}}\right|_{k=a H}
$$

For the spectral index of the scalar perturbations, we obtain in a similar way

$$
\begin{aligned}
& n_{S}(k)-1=\frac{d \ln \mathcal{P}_{\mathcal{R}}(k)}{d \ln k} \\
& =\frac{M_{P}^{2} W^{\prime}}{\frac{3}{2} U^{2}-W}\left(3 \frac{W^{\prime}}{W}-2 \frac{W^{\prime \prime}}{W^{\prime}}\right)=\left.\frac{4 \dot{H}-\frac{H \ddot{H}}{\dot{H}}}{\dot{H}+H^{2}}\right|_{k=a H} .
\end{aligned}
$$

On the basis of these relations, one can evidently obtain expressions for the runaway of spectral indices.

A procedure similar to that of calculating the power spectrum of scalar perturbations makes it possible to derive an exact expression for the power spectrum of tensor perturbations $\mathcal{P}_{\mathcal{G}}(k)$,

$$
\mathcal{P}_{\mathcal{G}}(k)=\frac{W}{6 \pi^{2} M_{P}^{4}}=\left.\frac{H^{2}}{2 \pi^{2} M_{p}^{2}}\right|_{k=a H},
$$

and to write down a formula for the spectral index of tensor perturbations:

$$
n_{G}=M_{P}^{2} \frac{\left(W^{\prime}\right)^{2}}{W\left(\frac{3}{2} U^{2}-W\right)}=\left.\frac{2 \dot{H}}{\dot{H}+H^{2}}\right|_{k=a H} .
$$

Let us note a specific result for the ratio of the spectral indices of tensor and scalar perturbations:

$$
r:=\frac{n_{G}}{n_{S}}=2 \dot{H}\left[5 \dot{H}+H^{2}-\frac{H \ddot{H}}{\dot{H}}\right]^{-1} .
$$

Since the improvement in Eqs. (7) and (9) consists in that the denominator contains $\left(\dot{H}+H^{2}\right)$ rather than $H^{2}$, the improvement does not manifest itself in this ratio.

In what follows, we will consider the tensor-toscalar ratio for squared amplitudes [2]

$$
\frac{T}{S}=\frac{A_{T}^{2}}{A_{S}^{2}}=-4 \frac{\dot{H}}{H^{2}}
$$

One can determine the amplitude of the tensor mode and estimate its contribution to the CMB anisotropy on the basis of the tensor-to-scalar ratio and the observed contribution of the scalar mode.

\section{Post-inflationary evolution of cosmological perturbations}

Consider the sequence of events at transition from the inflationary stage to the radiation and matter domination stages.

The inflationary stage is completed by scalar field decay and particle production, followed by nucleosynthesis and further evolution according to the standard scenario. Cosmological perturbations of different wavelengths (with different wavenumbers $k$ ) become classical quantities during several e-folds after the instant of coming outside the horizon. This time is designated as $t_{*}$.

Having crossed the horizon, the cosmological perturbations remain "frozen" in the gravitational background and do not change their magnitude in the comoving reference frame. The magnitude of perturbations evolves in a known way in the radiation-dominated epoch at entering under the horizon, an we denote this time as $t_{\mathrm{pr}}$.

The theory of cosmological perturbations is considered to be applicable in the initial epoch which begins before the cosmological scale of interest enters the horizon. This initial epoch begins much later than the nucleosynthesis, and therefore the matter constituents of the Universe, except nonbaryonic dark matter, are known. It has been established theoretically how perturbations of all components of the Universe evolve after the initial epoch evolve if the energy density of each component at that time is known.

The initial perturbation spectrum can be constructed using the transfer function from vacuum fluctuations at the instant $t_{*}$. It is known how to 
calculate this function; in doing so, the perturbation of each component is calculated from the curvature perturbation $\mathcal{R}_{\mathbf{k}}$ with the aid of the transfer function:

$$
g_{\mathbf{k}}(t)=T_{g}(t, k) \mathcal{R}_{\mathbf{k}},
$$

where $\mathcal{R}_{\mathbf{k}}(t)$ is determined at the time $t_{*}$ :

$$
\mathcal{R}_{\mathbf{k}}=-\left[\frac{H}{\dot{\phi}} \delta \phi_{k}\right]_{t=t_{*}} .
$$

Our improvement is associated with calculating the curvature perturbation on the basis of exact solutions to the Einstein equations (in the zero-order approximation) without using a slow-roll regime in this situation. Since we have obtained exact expressions for the cosmological parameters at an exit outside the horizon, we need, for confrontation with the observational data, to carry out a recalculation of the cosmological parameters to the present epoch. To do that, let us consider the post-inflationary evolution of the cosmological perturbations and find correction to the cosmological parameters.

It should be noted that, in the inflationary models under study, there is no natural exit from inflation. We therefore suppose that, after scalar field decay, the gravitational field turns into Friedmann radiation and matter-dominated stages during the same e-folds, characterized by the time $t_{*}$.

We use the standard method of re-calculating cosmological perturbations to Friedmann epochs. It is well known [5] that, during the radiationdominated stage $\left(a \sim t^{n}, n=1 / 2\right)$, and the matter-dominated stage $\left(a \sim t^{n}, n=2 / 3\right)$, the gravitational perturbations (the gravitational potential $\Phi_{k}$ ) are transformed in the following way:

During the radiation-dominated stage,

$\Phi_{\mathrm{k}}=\frac{2}{3} \mathcal{R}_{\mathrm{k}}$.

During the matter-dominated stage,

$$
\Phi_{\mathrm{k}}=\frac{3}{5} \mathcal{R}_{\mathrm{k}} \text {. }
$$

We conclude from these relations that the evolution of perturbations at superhorizon scales is reduced to simply rescaling their amplitudes.

Thus the power spectrum of the gravitational perturbations at the matter-dominated stage is determined through the power spectrum of curvature perturbations:

$$
\mathcal{P}_{\Phi}(M D)=\frac{9}{25} \mathcal{P}_{\mathcal{R}}
$$

This, in turn, allows us to determine the density contrast and the power spectrum of scalar perturbations:

$$
\begin{gathered}
\delta_{\mathbf{k}}=\frac{2}{3}\left(\frac{k}{a H}\right)^{2} T_{g}(t, k) \Phi_{\mathbf{k}}, \\
\mathcal{P}_{\delta}(k, t)=\frac{4}{25}\left(\frac{k}{a H}\right)^{4} T_{g}(t, k)^{2} \mathcal{P}_{\mathcal{R}},
\end{gathered}
$$

which can be compared with the observational data.

In the case of arbitrary wavelengths of the perturbations, the transfer function can be calculated numerically. The values of the transfer function have been tabulated for many cosmological models.

Thus our improvement introduced at the afterinflationary stage leads finally to improved relations containing observational data. Let us apply the methods described to some exact solutions.

\section{Cosmological parameters for ex- act solutions}

\subsection{Power-law inflation.}

First of all, consider power-law inflation. The scale factor of such a model is

$$
a(t)=a_{s} t^{m}, \quad a_{s}=\text { const. }
$$

For the spectral indices of scalar and tensor perturbations we obtain

$$
n_{S}-1=\frac{2}{1-m}=n_{G} .
$$

If $m>1$ and $\left|n_{S}-1\right|<0.2$, we get a reasonable of the degree of expansion: $m>9$.

The tensor-to-scalar spectral index ratio

$$
r=\frac{2}{3-m}
$$

is critically connected with the value $m=3$. When $m=3$, we have $r \rightarrow \infty$. For $1 \leq m<3$, we have $1 \leq r<\infty$, while for $m>3$ we have $-\infty<r<0$. When $m \geq 9$, we obtain $r \geq-1 / 3$, i.e. $|r|<$ 0.333 , and as $m$ grows, the spectral index of tensor perturbations becomes smaller and smaller as compared with the scalar one. One should note that these results are distinct from those of [6], which is caused by an error in the expression for the spectral index of scalar perturbations, Eq. (17) of the cited paper. 


\subsection{De Sitter solutions}

The de Sitter solution describes Universes with and without coordinate singularities, with the scale factor

$$
a(t)=a_{s} \sinh \left(h_{*} t\right), \quad a_{s}=\text { const },
$$

and

$$
a(t)=a_{s} \cosh \left(h_{*} t\right),
$$

respectively. The spectral indices and their ratio are calculated using the general formulas and have the following form:

For a "singular" Universe,

$$
\begin{aligned}
& n_{S}-1=\frac{2\left[2+\cosh ^{2}\left(h_{*} t\right)\right]}{\sinh ^{2}\left(h_{*} t\right)} \\
& n_{G}=-\frac{2}{\sinh ^{2}\left(h_{*} t\right)}, \quad r=\frac{2}{5+\cosh ^{2}\left(h_{*} t\right)} ; \\
& \mathcal{P}_{\mathcal{R}}=\frac{\operatorname{coth}^{4}\left(h_{*} t\right)}{8 M_{p}^{2} h_{*}\left[1-\operatorname{coth}^{2}\left(h_{*} t\right)\right]} \\
& \mathcal{P}_{\mathcal{G}}=\frac{\operatorname{coth}^{2}\left(h_{*} t\right)}{2 \pi^{2} M_{p}^{2}}, \quad \frac{T}{S}=4 \frac{h_{*}\left[1-\operatorname{coth}^{2}\left(h_{*} t\right)\right]}{\operatorname{coth}^{2}\left(h_{*} t\right)}
\end{aligned}
$$

In the case of the nonsingular de Sitter solution,

$$
\begin{aligned}
& n_{S}-1=\frac{2\left[2-\sinh ^{2}\left(h_{*} t\right)\right]}{-\cosh ^{2}\left(h_{*} t\right)}, \\
& n_{G}=\frac{2}{\cosh ^{2}\left(h_{*} t\right)}, \quad r=\frac{2}{5-\sinh ^{2}\left(h_{*} t\right)}, \\
& \mathcal{P}_{\mathcal{R}}=\frac{\tanh ^{4}\left(h_{*} t\right)}{8 M_{p}^{2} h_{*}\left[1-\tanh ^{2}\left(h_{*} t\right)\right]}, \\
& \mathcal{P}_{\mathcal{G}}=\frac{\tanh ^{2}\left(h_{*} t\right)}{2 \pi^{2} M_{p}^{2}} \\
& \frac{T}{S}=4 \frac{h_{*}\left[1-\tanh ^{2}\left(h_{*} t\right)\right]}{\tanh ^{2}\left(h_{*} t\right)}
\end{aligned}
$$

The scalar field must be imaginary.

\subsection{Generalized exponential inflation}

Consider a scale factor which is defined as

$$
a=a_{s} e^{\left(A e^{\lambda t}+B t\right)} \text {. }
$$

The Hubble parameter is

$$
H(t)=A \lambda e^{\lambda t}+B .
$$

Let us calculate the spectral indices of scalar and tensor modes of perturbation as well as the power spectra:

$$
\begin{aligned}
& n_{S}-1=\frac{4 A \lambda e^{\lambda t}-\lambda\left(A \lambda e^{\lambda t}+B\right)}{A \lambda^{2} e^{\lambda t}+\left(A \lambda e^{\lambda t}+B\right)^{2}} \\
& n_{G}=\frac{2 A \lambda^{2} e^{\lambda t}}{A \lambda^{2} e^{\lambda t}+\left(A \lambda e^{\lambda t}+B\right)^{2}} \\
& \mathcal{P}_{\mathcal{R}}=\frac{\left(A \lambda e^{\lambda t}+B\right)^{4}}{8 A \lambda^{2} M_{p}^{2} e^{\lambda t}}, \quad \mathcal{P}_{\mathcal{G}}=\frac{\left(A \lambda e^{\lambda t}+B\right)^{2}}{2 \pi M_{p}^{2}} .
\end{aligned}
$$

The ratio of the spectral indices and the tensorto-scalar ratio at crossing of Hubble radius are

$$
\begin{aligned}
r & =\frac{A \lambda^{2} e^{\lambda t}}{5 A \lambda^{2} e^{\lambda t}+\left(\lambda e^{\lambda t}+B\right)^{2}-\lambda\left(\lambda e^{\lambda t}+B\right)}, \\
\frac{T}{S} & =4 \frac{A \lambda^{2} e^{\lambda t}}{\left(A \lambda e^{\lambda t}+B\right)^{2}} .
\end{aligned}
$$

\subsection{Exponential-power-law inflation.}

In this model, the evolution of the scale factor is defined as

$$
\begin{aligned}
& a(t)=a_{s} t^{n} \exp \left(h_{*} t\right), \\
& n=A^{2}\left(2 M_{P}^{2}\right), \quad a_{s}=\text { const. }
\end{aligned}
$$

The cosmological parameters are, in this case, written as

$$
\begin{aligned}
& n_{S}-1=\frac{2\left(h_{*} t-n\right)}{\left(h_{*} t+n\right)^{2}-n}, \\
& n_{G}=\frac{2 n}{n-\left(h_{*} t+n\right)^{2}} \\
& \mathcal{P}_{\mathcal{R}}=\frac{t^{4}}{8 M_{p}^{2}\left(h_{*} t+n\right)^{2}\left[1-t\left(h_{*} t+n\right)\right]}, \\
& \mathcal{P}_{\mathcal{G}}=\frac{t^{2}}{2 \pi^{2} M_{p}^{2}\left(h_{*} t+n\right)^{2}} .
\end{aligned}
$$

The ratio of the spectral indices and the tensor-toscalar ratio are again easily calculated:

$$
\begin{aligned}
& r=\frac{2 n}{\left(h_{*} t+n\right)^{2}+2 h_{*} t-3 n}, \\
& \frac{T}{S}=4 \frac{t^{2}}{1-t\left(h_{*} t+n\right)} .
\end{aligned}
$$

Let us find the cosmological parameters for scale factor evolution

$$
a=a_{s} e^{\lambda\left(t-t_{0}\right)^{\mu}} .
$$


By calculations similar to the previous ones, we obtain:

$$
\begin{aligned}
& n_{S}-1=\frac{3 \mu+1}{\mu\left(t-t_{0}\right)^{\mu}+\mu-1}, \\
& n_{G}=\frac{2}{1+[\mu /(\mu-1)]\left(t-t_{0}\right)^{\mu}}, \\
& \mathcal{P}_{\mathcal{R}}=\frac{\lambda^{3} \mu^{3}}{8 M_{p}^{2}} \frac{\left(t-t_{0}\right)^{3 \mu-2}}{\mu-1}, \\
& \mathcal{P}_{\mathcal{G}}=\frac{1}{2}\left(\frac{\mu \lambda}{M_{p}^{2}}\right)^{2}\left(t-t_{0}\right)^{2 \mu-2}, \\
& r=\frac{2 \lambda t(\mu-1)}{5 \lambda \mu(\mu-1)+\lambda^{2} \mu^{2} t^{\mu+1}-\mu+2}, \\
& \frac{T}{S}=\frac{4(\mu-1)}{\mu \lambda\left(t-t_{0}\right)^{\mu}}
\end{aligned}
$$

In these models, the values of the constants are restricted by $T / S<0.2$ according to the observational data 2 .

\section{Discussion}

Further developing the exact methods of obtaining the key cosmological parameters, suggested in [4, 6], we have presented exact formulas for the power spectra of scalar and tensor (gravitational) perturbations and the corresponding spectral indices. (From the formulas presented, it is easy to determine the runaway of the spectral indices and their form for the models enumerated in this article. These results will be given elsewhere.)

Let us again emphasize the simplicity of the method and its applicability to any models in which the gravitational field of the evolving Universe or the total energy potential are known (see $[4,6]$ ).

\section{Acknowledgment}

The authors thank the participants of the SchoolSeminar "Modern Theoretical Problems of Gravitation and Cosmology" GRACOS-2007, especially V. N. Lukash and A. A. Starobinsky, for a discussion of the results and a productive debate.

\section{References}

[1] A. A. Starobinsky, Phys. Lett. 918B, 24 (1980);

A. H. Guth, Phys. Rev. D 23, 347 (1981);

A. D. Linde, Phys. Lett. 108B, 389 (1982);

A. Albrecht and P. J. Steinhardt, Phys. Rev. Lett. 48, 1220 (1982).
[2] V. N. Lukash, Uspekhi Fiz. Nauk 176, 113 (2006).

[3] A. R. Liddle and D. H. Lyth, Phys. Rep. 231, 1 (1993).

[4] S. V. Chervon, Gen. Rel. Grav. 36, 1547 (2004).

[5] A. Riotto, Inflation and the Theory of Cosmological Perturbations, hep-ph/0210162.

[6] S. V. Chervon, M. Novello and R. Triay, Grav. \& Cosmol. 11, 329 (2005).

[7] S. V. Chervon, Exact inflation and the key cosmological parameters. In: Proc. of the Russian School and International Conference on Gravitation, Cosmology and Relativistic Astrophysics GRACOS-2007, 09-16 September 2007, TGGPU, Kazan-Yalchik, 2007, pp. 174-178;

S. V. Chervon, Exact inflation and cosmological parameters, Issledovano $\mathrm{v}$ Rossii (electronic journal), No. 100, pp. 1035-1040 (2007); http://zhurnal.ape.relarn.ru/articles/2007/100.pdf

[8] S. V. Chervon, Nonlinear Fields in the Theory of Gravitation and Cosmology (Middle-Volga Scientific Center, Ulyanovsk State University, 1997).

[9] S. V. Chervon, V. M. Zhuravlev, and V. K. Shchigolev, Phys. Lett. 398B, 269 (1997).

[10] G. F. R. Ellis and M. S. Madsen, Class. Quantum Grav. 8, 667 (1991).

[11] J. D. Barrow, Phys. Rev. D 49, 3055 (1994).

[12] R. Maartens, D. R. Taylor and N. Roussos, Phys. Rev. D 52, 3358 (1995).

[13] V. M. Zhuravlev and S. V. Chervon, JETP 118, 259 (2000).

[14] A. H. Guth, Inflation, astro-ph/0404546.

[15] E. D. Stewart, The spectrum of density perturbations produced during inflation to leading order in a general slow-roll approximation, astro-ph/0110322.

[16] P. Binetruy and M. K. Gaillard. Phys. Rev. D 34, 3069 (1986). 